\title{
Entropies and Equilibria of Many-Particle Systems: An Essay on Recent Research
}

\author{
A.Arnold* \\ J.A. Carrillo ${ }^{\dagger}$ \\ L. Desvillettes ${ }^{\ddagger}$ \\ J.Dolbeault ${ }^{\S}$ \\ A. Jüngel \\ C. Lederman" \\ P.A. Markowich** \\ G. Toscani ${ }^{\dagger \dagger}$ \\ C. Villani ${ }^{\ddagger \ddagger}$
}

This essay is intended to present a fruitful collaboration which has developed among a group of people whose names are listed above: entropy methods have proved over the last years to be an efficient tool for the understanding of the qualitative properties of physically sound models, for accurate numerics and for a more mathematical understanding of nonlinear PDEs. The goal of this essay is to sketch the historical development of the concept of entropy in connection with PDEs of continuum mechanics, to present recent results which have been obtained by the members of the group and to emphasize the most striking achievements of this research. The presentation is by no way an exhaustive review of the methods and results involving the entropy, not even in the field of PDEs. Many other researchers in and outside Europe have contributed to the development of this field, including - but not only in collaboration with some of the people of the group. However, it can be claimed that this group had a leading role over the recent years and this essay is intended to explain how this occurred.

\section{Motivation and Applications}

In the fast moving development of new technologies, ranging from microelectronics to space crafts, applied mathematics plays a substantial role in two fundamental steps of the realization process: modeling and numerical simulation. These two issues are closely connected, and vital to continuously improve the physical description of the relevant phenomena. In many novel applications the modeling involves the knowledge of the behavior of systems composed of a large number of interacting particles: electrons in micro-devices, ions in the

\footnotetext{
*Institut für Numerische Mathematik, Universität Münster, D-48149 Münster, Germany

†ICREA and Departamento de Matemática, Universidad Autonoma de Barcelona, 08193-Bellaterra, Spain.

${ }^{\ddagger}$ Ecole Normale Supérieure de Cachan, 61, avenue du Président Wilson 94235 Cachan, France.

$\S$ Ceremade, Université Paris IX Dauphine, Place de Lattre de Tassigny F-75775 Paris, France.

『Fachbereich Mathematik und Informatik, Universität Mainz, Germany.

"Departamento de Matemática, Universidad de Buenos Aires, 1428 Buenos Aires, Argentina.

** Department of Mathematics, University of Vienna, Boltzmanngasse 9, Vienna, Austria.

${ }^{\dagger \dagger}$ Department of Mathematics, University of Pavia, via Ferrata 1, 27100 Pavia, Italy.

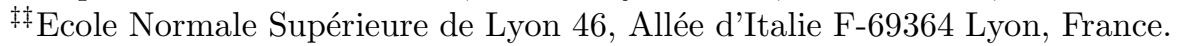


plasma of fusion reactors, atoms in a Bose-Einstein condensate, gas flowing over the wings of aircrafts, etc. One of the main features of such systems is their tendency (if left alone) to converge to an equilibrium configuration as time becomes large (usually this time is rather small viewed at our macroscopic scale). This is even part of our daily experience at a macroscopic scale: whenever we create a breeze in a room by opening a window, after shutting it again the gas will come to rest in a very short time.

Very often, there is a thermodynamical principle underlying this property of trend to equilibrium: as time progresses, interactions between particles lead to the increase of a distinguished functional called entropy (second law of thermodynamics, formulated at the end of the nineteenth century). Gibbs' principle asserts that the equilibrium distribution is the one which achieves the maximum entropy under the constraints imposed by the conservation laws. On the other hand, when such a large particle system is subjected to a continuous exterior stimulus (e.g. a force field) it exhibits an interplay between non-equilibrium and equilibrium regimes, which are of particular interest in computational physics and scientific computing: E.g., the simulation of the air flow around a space capsule at re-entry into the atmosphere requires to combine fluid mechanical equations for the equilibrium regions with finer so-called kinetic models for the non-equilibrium domains. Such kinetic equations provide a very accurate description of reality by modeling the collisional particle interactions in the position and momentum phase space. However, since they are often prohibitively expensive for numerical simulations of real three-dimensional problems, one frequently resorts to fluid dynamical equations, which only use position (and of course time) as independent variables. But the finer kinetic description is still crucial to understand the range of validity of the simplified models.

Since a system in (local) equilibrium depends only on a limited number of unknowns certain macroscopic observables- both the identification of equilibrium configurations and the speed of convergence towards equilibrium are of paramount importance for applications. Knowing this convergence rate is the only way to understand the relevant time scale for the equilibration process, which in turn is important for modeling and hence for guessing the feasibility of numerical simulations. Driven by technological needs in semiconductor development, plasma physics, and aeronautics the mathematical research of equilibration processes in the kinetic theory of rarefied gases recently experienced an unprecedented thrust.

The Boltzmann equation is the most famous kinetic model, both due to its important current applications and for historical reasons: For this model L. Boltzmann proved his celebrated $H$-theorem about the increase of the entropy ${ }^{1}$, which was the first analytical proof ever of the second principle of thermodynamics. Boltzmann's analysis can be adapted for numerous variants of the Boltzmann equation which are used in the description of collective dynamics in physics, engineering and biology, and in the description of the temporal transition from non-equilibrium to equilibrium in thermodynamical processes.

This report describes a collaborative research program, carried out by the research groups of the authors, partly in cooperation with other applied mathematicians. In part already known methods and partly the development of new analytical techniques to calculate the

\footnotetext{
${ }^{1}$ L. Boltzmann, Weitere Studien über das Wärmegleichgewicht unter Gasmolekülen. Sitzungsberichte der Akademie der Wissenschaften 66, (1872), 275-370; Lectures on Gas Theory. University of California Press, Berkeley, 1964. Translated by S.G. Brush. Reprint of the 1896-1898 Edition. Reprinted by Dover Publications, 1995.
} 
(optimal) speed of convergence towards equilibrium for both nonlinear kinetic equations and diffusion equations (obtained by scaling limit techniques from kinetic models) were necessary.

As a result of this recent research work, several unexpected links between the study of entropy production in nonlinear many-particle systems and the field of functional inequalities were unreaveled. Thus, the findings have important implications for related problems, mainly in the modeling of diffusion processes. These evolution processes have a variety of applications in physics and in industry. Among other things, our results are important for the validation of diffusion processes modeling heat transport, porous media flows, composite materials manufacturing, fast diffusion (Okuda-Dawson diffusion of plasma, solid state physics), thin films, liquid films, and moving contact lines, which are of basic importance in video tape production, cooling devices and biomedical devices.

A good part of the results of this joint work, which are collected at the end of this report, can be found in the corresponding preprints in the Preprint Archive, http://hyke.org.

\section{From Boltzmann's $H$-Theorem to entropy methods for partial differential equations}

For a better understanding of the main ideas which are at the basis of our studies, we briefly recall previous applications of entropy to sciences.

Since its introduction by Boltzmann more than one hundred years ago, the notion of entropy has been widely used in the study of dissipating systems, both by physicists and by mathematicians. It has also gained its way in engineering applications via the important concept of information developed by Shannon ${ }^{2}$, and in statistics after the invention of Fisher's information ${ }^{3}$.

Different applications of the concept of entropy have been introduced by $\mathrm{Lax}^{4}$ for hyperbolic systems of conservation laws, and by DiPerna ${ }^{5}$ in the framework of compensated compactness. Also, the well-celebrated theorem of DiPerna and P.-L. Lions ${ }^{6}$ on global existence of renormalized solutions of the Boltzmann equation, has a proof that is strongly based on the use of Boltzmann's entropy. Another, not so well-known example is the use of the entropy in the famous work of $\mathrm{Nash}^{7}$ on the regularity of the solution of certain diffusion processes. In all these works, the entropy is introduced for a purpose which is not its original one, but appears surprisingly useful.

\footnotetext{
${ }^{2}$ C. E. Shannon, Collected papers. Edited by N. J. A. Sloane and Aaron D. Wyner. IEEE Press, New York, 1993; T.M. Cover and J.A. Thomas, Elements of Information Theory. J. Wiley \&amp; Sons Inc., New York, 1991.

${ }^{3}$ R. Fisher, Theory of statistical estimation. Math. Proc. Cambridge Philos. Soc. 22 (1925), 700-725.

${ }^{4}$ P.D. Lax, Hyperbolic systems of conservation laws and the mathematical theory of shock waves. Conference Board of the Mathematical Sciences Regional Conference Series in Applied Mathematics, 11. Society for Industrial and Applied Mathematics, Philadelphia, Pa., (1973), v+48.

${ }^{5}$ R.J. DiPerna, Compensated compactness and general systems of conservation laws. Trans. Amer. Math. Soc. 292 (1985), 383-420.

${ }^{6}$ R.J. DiPerna and P.-L. Lions, On the Cauchy problem for the Boltzmann equation: Global existence and weak stability. Ann. of Math. (2) 130 (1989), 312-366.

${ }^{7}$ J. Nash, Continuity of solutions of parabolic and elliptic equations. Amer. J. Math. 80 (1958), 931-954.
} 
Twenty years ago, Cercignani ${ }^{8}$ had stated a conjecture about Boltzmann's entropy production which would imply an exponentially fast trend to equilibrium, but there was no clue of the proof of this conjecture. We remark once again that the verification of this conjecture is not merely an academic exercise, but on the contrary it has e.g. extremely important consequences in numerical simulation of re-entry problems, since it provides a theoretical justification of the use of fluid dynamics in the modeling. In 1989 Desvillettes $^{9}$ found an interesting lower bound for the entropy production relative to the Boltzmann equation. However, this lower bound was not precise enough to settle the Cercignani conjecture.

At the beginning of the nineties, Carlen and Carvalho ${ }^{10}$ managed to establish a more precise lower bound. More importantly, they pointed out some connections of Cercignani's conjecture with the field of information theory and some functional inequalities (so-called logarithmic Sobolev inequality) which had become famous over the last decades for their appearing in various branches of modern mathematics ${ }^{11,12}$. An equivalent inequality had been shown a long time ago by Stam, working in information theory ${ }^{13}$.

The Gross logarithmic Sobolev inequality was introduced by Toscani ${ }^{14}$ into the theory of kinetic Fokker-Planck diffusion processes. His work suggested a more physical way of understanding the Bakry-Emery approach (originally developed in probabilistic diffusion theory), in terms of the physical entropy production and production of entropy production. This was at the beginning of the year 1997. Main problems were still mostly open then, but the kinetic community in Europe was beginning to be widely interested in these topics.

\section{Entropy methods}

The last years have seen the rapid development of so-called entropy methods for the study of convergence to equilibrium in many-particle systems, and have led to the solution of most of the problems stated above.

The main idea behind entropy methods is to establish quantitative variants of the mechanism of increase of the entropy. This approach has the merit to stand upon a clear physical basis, and experience has shown its robustness and flexibility. The by now standard strategy can be summed up in four steps:

- Step 1 : Identify the equilibrium state and the entropy functional, or more generally Lyapunov functional, $E$, which is associated to the equation. In many cases, the

${ }^{8} \mathrm{C}$. Cercignani, $H$-theorem and trend to equilibrium in the kinetic theory of gases. Arch. Mech. $\mathbf{3 4}$ (1982), 231-241.

${ }^{9}$ L. Desvillettes, Entropy production rate and convergence in kinetic equations. Comm. Math. Phys. 26 (1989), 687-702.

${ }^{10}$ E.A. Carlen and M. Carvalho, Strict entropy production bounds and stability of the rate of convergence to equilibrium for the Boltzmann equation. J. Statist. Phys. 67 (1992), 575-608.

${ }^{11}$ L. Gross, Logarithmic Sobolev inequalities. Amer. J. of Math. 97 (1975), 1061-1083

${ }^{12}$ D. Bakry and M. Emery, Diffusions hypercontractives. In Sém. Proba. XIX, 1123 Lecture Notes in Math. Springer, 1985, 177-206.

${ }^{13}$ A. Stam, Some inequalities satisfied by the quantities of information of Fisher and Shannon. Inform. Control 2 (1959), 101-112.

${ }^{14}$ G. Toscani, Sur l'inégalité logarithmique de Sobolev. C.R. Acad. Sc. Paris 324 (1997), 689-694. 
derivation of this Lyapunov functional is quite standard ${ }^{15}$.

- Step 2 : Given the entropy (Lyapunov) functional $E$, attaining its maximum at the equilibrium $f_{\infty}$, the discrepancy between a distribution function $f$ and the equilibrium $f_{\infty}$ is in this approach measured by $E\left[f \mid f_{\infty}\right]=E\left(f_{\infty}\right)-E(f)$, thereafter called relative entropy. Often it is not advisable to try directly to prove that $f(t)$ converges to $f_{\infty}$, but rather to show that $E(f(t))$ converges to $E\left(f_{\infty}\right)$, which will be called "convergence in relative entropy".

- Step 3 : One considers as a main object of study the "entropy production functional" $P$, which is just the time-derivative of the entropy, $P(f(t))=d E(f(t)) / d t$.

- Step 4 : One tries to quantify the following idea: if, at some given time $t, f(t)$ is far from $f_{\infty}$, then $E(f(t))$ will increase notably at later times.

When trying to implement the preceding general principles, one can succeed in proving an entropy-entropy production inequality: this is a functional type inequality of the type $P(f) \geq \Theta\left(E\left[f \mid f_{\infty}\right]\right)$, where $H \rightarrow \Theta(H)$ is some continuous function, strictly positive when $H>0$. The main idea is that "entropy production controls relative entropy". Such an inequality implies an immediate solution to the problem of trend to equilibrium. Indeed, it implies a closed differential equation on the relative entropy, of the type $-(d / d t) E\left[f \mid f_{\infty}\right] \geq \Theta\left(E\left[f \mid f_{\infty}\right]\right)$. Usual techniques for ordinary differential equations (inequalities) imply that $E\left(f(t) \mid f_{\infty}\right)$ converges to 0 as $t \rightarrow \infty$. Moreover (and this is the crucial point for getting relevant results), if the function $\Theta$ is known with enough details, then one can compute an explicit rate of convergence. Thus, $\Theta(H)=\lambda H$ ("linear bound") implies exponential convergence, with speed given by the constant $\lambda$, while $\Theta(H)=K H^{1+\alpha}$ ("polynomial bound") implies polynomial rate of convergence, the relative entropy decaying at least like $t^{-1 / \alpha}$.

\section{Results}

The entropy method described in the previous section has been developed by the research groups of the authors from 1997 on, starting with classical equations in kinetic theory. From 1998 on, the partners collaborated in the TMR European Network Project "Asymptotic Methods in Kinetic Theory" (http://www.math.tu-berlin.de/ ${ }^{\sim}$ tmr). Their studies led to the following results:

1) the discovery of simple and sharp lower bounds for the entropy production of classical equations of kinetic theory such as Boltzmann's (settling the Cercignani conjecture, as we shall explain);

2) a very complete understanding of the entropy production method, which was generalized in an unexpected way to apply to a large family of equations, including nonlinear diffusion processes, and also mean-field diffusive equations. Moreover, the method was linked

\footnotetext{
${ }^{15}$ G.Toscani, Remarks on entropy and equilibrium states Appl. Math. Letters, 12 (1999) 19-25; J.A. Carrillo, A. Juengel, P. Markowich, G. Toscani and A. Unterreiter, Entropy production methods for degenerate parabolic problems and generalized Sobolev inequalities. Monatsh.Math. 133 (2001) 1-82.
} 
to the field of mass transportation; this field is well-known in probability theory ${ }^{16}$, with applications to economy (going back to the Nobel-prize winner Kantorovich). The Bakry-Emery method was re-interpretated, in a very surprising way, in terms of mass transportation, which enabled its generalization to complicated models such as granular flows;

3) a new way of studying time-intermediate asymptotic for equations which do not possess a steady state, but have certain self-similarity properties (such cases occur very frequently in applications);

4) theoretical results, in the form of new functional inequalities, which came as a byproduct of this study, even if this was not the main goal of the researchers.

\subsection{Entropy production functionals of Boltzmann and Landau.}

The results of this section have immediate applications to the development of numerical codes in rarefied gas dynamics and plasma physics. Moreover, they close a longstanding open problem in kinetic theory. When grazing collisions in the Boltzmann operator prevail, the evolution of the density in a dilute plasma is driven by the Landau operator ${ }^{17}$. An almost complete study of the entropy production functional of the Landau equation was performed $^{18}$. By using the logarithmic Sobolev inequality Desvillettes and Villani succeeded in proving Cercignani's conjecture for an over-Maxwellian cross section. Also "sharp" entropy production bounds for the Boltzmann collisional operator were obtained ${ }^{19}$. Physically speaking, it was proved that Cercignani's conjecture ${ }^{20}$ is "almost" true in the following sense: One can choose $\Theta(H)=$ const. $H^{1+\epsilon}$, with $\epsilon>0$ as small as desired, provided that $f$ decays fast enough and satisfies a Gaussian lower bound estimate. The assumption of positive values for $\epsilon$ can not be avoided; there are counterexamples. The case of decay to equilibrium for both Boltzmann and Landau equation with soft interaction and smoothed kernel ${ }^{21}$ was subsequently treated. Another application of Lyapunov functionals was found ${ }^{22}$. Recent applications of the entropy methods ${ }^{23}$ have been discussed.

\footnotetext{
${ }^{16}$ S.T. Rachev and L. Rschendorf, Mass Transportation Problems. Vol. II. Applications. Probability and its Applications, Springer-Verlag, New York, 1998.

${ }^{17}$ L. Landau, Die kinetische Gleichung für den Fall Coulombscher Wechselwirkung. Phys. Z. Sovjet. 10 (1936), 154.

${ }^{18}$ L. Desvillettes and C. Villani, On the Spatially Homogeneous Landau Equation for Hard Potentials. Part II: H-Theorem and Applications. Comm. Partial Differential Equations 25, n. 1-2, (2000), $261-298$.

${ }^{19}$ G. Toscani and C. Villani, Sharp entropy production bounds and explicit rate of trend to equilibrium for the spatially homogeneous Boltzmann equation. Commun. Math. Phys. 203, (1999) 667-706.

${ }^{20} \mathrm{C}$. Cercignani, $H$-theorem and trend to equilibrium in the kinetic theory of gases. Arch. Mech. $\mathbf{3 4}$ (1982) 231-241.

${ }^{21} \mathrm{G}$. Toscani and C. Villani, On the trend to equilibrium for some dissipative systems with slowing increasing a priori bounds. J. Statist. Phys. 98 (2000) 1279-1309.

${ }^{22}$ G. Toscani and C. Villani, Probability metrics and uniqueness of the solution to the Boltzmann equation for a Maxwell gas. J. Statist. Phys. 94, (1999) 619-637.

${ }^{23}$ L. Desvillettes and C. Villani, Entropic Methods for the Study of the Long Time Behavior of Kinetic Equations. Proceedings of the 16-th ICTT Congress, Atlanta, (1999), to appear; (TMR Preprint Archive).
} 


\subsection{Linear Fokker-Planck type diffusion equations.}

A detailed analysis of entropy production inequalities for linear Fokker-Planck type equations was performed ${ }^{24}$. For this model, one can define a whole family of relative entropy functionals, of the form $H_{\Phi}\left(f \mid f_{\infty}\right)=\int \Phi\left(f / f_{\infty}\right) f_{\infty}$, whose "extremals" are given by $\Phi(h)=h \log h-h+1$ at one end, $\Phi(h)=(h-1)^{2} / 2$ at the other hand. For each of these entropies one can perform a Bakry-Emery type argument to prove logarithmic Sobolev type inequalities; and they are all the stronger as the nonlinearity in the relative entropy is weaker (the strongest one corresponding to the $h \log h$ nonlinearity). The implications of convergence in relative entropy have been established in great generality ${ }^{25}$. Moreover the interplay between the Fokker-Planck equation and the field of differential inequalities ${ }^{26}$ was systematically analyzed. This is a surprising situation where a physical system is used to prove a result in PDE's analysis!

\subsection{Non-linear diffusions of second order with potential confine- ment.}

Maybe the most important application of entropy production methods is linked to the largetime behavior of diffusion problems. For the first time entropy production for porous medium and fast diffusion equations with potential confinement was studied ${ }^{27}$. Subsequently ${ }^{28}$, nonlinear Fokker-Planck type equations and strongly coupled systems of degenerate nonlinear parabolic equations were analyzed. These equations arise in semiconductor theory, plasma physics, and stellar dynamics. The entropy production analysis was also extended to systems of degenerate parabolic equations arising in non-equilibrium thermodynamics, semiconductor energy-transport theory, and alloy solidification processes ${ }^{29}$. Additional applications include explicit rates of convergence towards the self-similar solution of both slow (porous medium) and fast diffusion equations. This is a famous problem which goes back to the seventies ${ }^{30}$. Very recently, nonlinear fast diffusion equations with finite equilibrium mass and infinite equilibrium entropies ${ }^{31}$ were atudied. The analysis of second order diffusion equation with

\footnotetext{
${ }^{24}$ J.A. Carrillo and G. Toscani, Exponential convergence toward equilibrium for homogeneous FokkerPlanck-type equations. Mathem. Methods Appl. Sciences 21 (1998) 1269-1286; A. Arnold, P. A. Markowich, G. Toscani and A. Unterreiter, On Convex Sobolev Inequalities and the Rate of Convergence to Equilibrium for Fokker-Planck Type Equations. Comm. Partial Differential Equations, 26 (2001), 43-100.

${ }^{25}$ A. Arnold, P.A. Markowich, G. Toscani and A. Unterreiter. On Generalized Csiszar-Kullback Inequalities. Monatsh. Math. 131 (2000), 235-253.

${ }^{26}$ P.A. Markowich and C. Villani, On the trend to equilibrium for the Fokker-Planck equation: an interplay between physics and functional analysis. Mat. Contemp. 19 (2000), 1-29.

${ }^{27}$ J. A. Carrillo and G. Toscani, Asymptotic $L^{1}$-decay of the porous medium equation to self-similarity. Indiana Univ. Math. J. 46 (2000), 113-142.

${ }^{28}$ J.A. Carrillo, A. Juengel, P. Markowich, G. Toscani and A. Unterreiter, Entropy production methods for degenerate parabolic problems and generalized Sobolev inequalities. Monatsh.Math., 133 (2001), 1-82.

${ }^{29}$ A. Juengel, P. Markowich and G.Toscani, Decay rates for solutions of degenerate parabolic systems. Electron. J. Diff. Eqs. Conf. 06 (2001), 189-202.

${ }^{30} \mathrm{~S}$. Kamenomostskaya, The asymptotic behavior of the solution of the filtration equation. Israel J. Math. 14 (1973), 76-87.

${ }^{31}$ C. Lederman and P.A. Markowich, On fast-diffusion equations with infinite equilibrium entropy and finite equilibrium mass. Comm. Partial Differential Equations 28 (2003), 301-332.; J.A. Carrillo, C. Lederman,
} 
potential confinement has been recently generalized to models of dissipating granular flows ${ }^{32}$. Further applications ${ }^{33}$ were also investigated.

\subsection{Non-linear diffusions of fourth order.}

Recent applications of the entropy production technique led to new and interesting results for fourth order diffusion equations. Mainly, two different physical models were treated. The first one is a nonlinear fourth-order parabolic equation arising in quantum semiconductor modeling. Exponential convergence to equilibrium in relative entropy in the one-dimensional case with no-flux boundary conditions ${ }^{34}$ has been shown. Secondly, a model arising in the field of the tension-dominated motion of a spreading droplet (thin-film equation) ${ }^{35}$ has been investigated.

\subsection{Mean field models.}

Many physical multi-particle models include force terms, in particular self-consistent effects described by mean field interactions. A very popular model from plasma physics is the Vlasov-Fokker-Planck equation, in which the collision operator is the Fokker-Planck operator, and the forces include both confinement and self-consistent interactions. If the interaction is of Coulomb type, then one speaks of the Vlasov-Poisson-Fokker-Planck model. Some of the authors introduced ${ }^{36}$ the entropy production method for drift-diffusion-Poisson equations and generalized the method to systems of two particle species ${ }^{37}$. Also more general nonlinear drift-diffusion systems with Poisson coupling ${ }^{38}$ were treated. These results are reminiscent of the techniques developed for nonlinear second-order diffusions without self-consistent interactions.

\subsection{Spatially inhomogeneous models}

Entropy production techniques have been applied to spatially inhomogeneous models in two situations. The algebraic decay towards the global equilibrium for the linear spatially in-

P.A. Markowich and G. Toscani, Poincaré Inequalities for Linearizations of Very Fast Diffusion Equations., Nonlinearity 15 (2001), 1-16.

${ }^{32}$ J.A. Carrillo, R.J. McCann, C. Villani, Kinetic equilibration rates for granular media and related equations: entropy dissipation and mass transportation estimates, Rev. Mat. Iberoamericana, 19, (2003) 1-48.

${ }^{33}$ A. Arnold and J. Dolbeault, Refined convex Sobolev inequalities. (TMR Preprint Archive), (2001).

${ }^{34}$ A. Juengel and G. Toscani, Exponential decay in time of solutions to a nonlinear fourth-order parabolic equation. in press on Z. Angew. Math. Phys. 54, (2003) 377-386

${ }^{35}$ J.A. Carrillo and G. Toscani, Large-time asymptotics for strong solutions of the thin film equation. Commun. Math. Phys. 225 (2002), 113-142.

${ }^{36}$ A. Arnold, P. A. Markowich, G. Toscani and A. Unterreiter, On Convex Sobolev Inequalities and the Rate of Convergence to Equilibrium for Fokker-Planck Type Equations. Comm. Partial Differential Equations 26 (2001) 43-100.

${ }^{37}$ A. Arnold, P. Markowich and G. Toscani, On large time asymptotics for drift-diffusion-Poisson systems. Transport Theory Statist. Phys. 29 (2000), no. 3-5, 571-581.

${ }^{38}$ P. Biler, J. Dolbeault and P. A. Markowich, Large Time Asymptotics of Nonlinear Drift-Diffusion Systems with Poisson Coupling. Transport Theory Statist. Phys., 30 (2001), 521-536 
homogeneous Fokker-Planck equation in a confining potential ${ }^{39}$. This is the first explicit estimate of convergence towards the equilibrium in the context of the spatially inhomogeneous kinetic theory. Subsequently, a large-time-analysis of spatially inhomogeneous discrete velocity models for kinetic initial-boundary value problems ${ }^{40}$. Exponential convergence to the equilibrium is obtained here via elaborate multiplier techniques, since the mass transport across the boundary is the main equilibrating effect.

\subsection{Steady-state mean field models.}

The existence of steady states for diffusive (elliptic) equations for systems with a (nonlinear) mean field term (electrostatic conductor and, respectively, gravitational stellar mean field models) was analyzed by bifurcation, variational and comparison techniques involving the entropy $^{41}$. The (singular) charge neutral (small Debye lenght) limit was also analyzed: for typical parameters ranges the problem is reduced to solve an obstacle problem, showing the occurrence of a depletion (vacuum) region ${ }^{41,42}$.

\section{$5 \quad$ Expected impacts}

Because of its clear physical basis, its robustness and flexibility, the entropy production method has generated much interest in the PDE community. Our studies of trend to equilibrium are mainly useful for problems in the modeling and numerical simulation of systems in which a thermodynamical principle prevails. This concerns in particular the technological development of microelectronics and nano-machines, models for systems in fluid dynamics, granular materials, plasma physics, stellar media and many others. The methods give information on the time scale for relaxation to equilibrium, and therefore on the qualitative behavior of the models as well as on their validity. The obtained results yield a new mathematical method which can be applied to a very large array of models. It is expected to become a standard tool of the analysis of evolution PDEs. In particular, the entropy production method helps in the rigorous derivation and validation of models and in computational physics, in particular for the justification of numerical schemes. Entropy production techniques are by now essential in computer simulation and modeling of diffusion processes, to justify the numerical passage from the mesoscopic level of description typical of kinetic equations towards the macroscopic equations of fluid dynamics. More than one century after its first introduction, entropy reveal itself to be a flexible and robust instrument both for

\footnotetext{
${ }^{39}$ L. Desvillettes and C. Villani, On the trend to global equilibrium in spatially inhomogeneous entropydissipating systems. Part I : The linear Fokker-Planck equation. Preprint n. 9918 of the CMLA, ENS de Cachan (1999). Comm. Pure Appl. Math., 54 (2001) 1-42.

${ }^{40}$ A. Arnold, J.A. Carrillo and M.D. Tidriri, Large-time behavior of discrete kinetic equations with nonsymmetric interactions. Math. Models Methods Appl. Sci. 12 (2002) 1555-1564

${ }^{41}$ L. Caffarelli, J. Dolbeault, P. Markowich and C. Schmeiser, On Maxwellian Equilibria of Insulated Semiconductors. Interfaces Free Bound., 2 (2000), n. 3, 331-339; P. Biler, J. Dolbeault, M. Esteban, P. Markowich and T.Nadzieja, Steady states for Streater's energy-transport models of self-gravitating particles. Preprint Ceremade n. 0039 (2000) (TMR Preprint Archive).

${ }^{42}$ J. Dolbeault, P.A. Markowich and A. Unterreiter, On Singular Limits of Mean-Field Equations. Arch. Ration. Mech. Anal., 158 (2001), 319-351.
} 
theory and applications.

Acknowledgment: The authors acknowledge financial support both from the TMR project "Asymptotic Methods in Kinetic Theory", contract No. ERBFRMXCT 970157, and from the HYKE project "Hyperbolic and Kinetic Equations: Asymptotics, Numerics, Applications", contract No. HPRN-CT-2002-00282, funded by the EC. The work of Peter Markowich was supported by the Austrian Science Fund FWF through his Wittgenstein 2000 Award and through the project P14876 entitled "Fokker-Planck und Mittlere-Feld-Gleichungen". 